Article

\title{
Development of 65 Novel Polymorphic cDNA-SSR Markers in Common Vetch (Vicia sativa subsp. sativa) Using Next Generation Sequencing
}

\author{
Jong-Wook Chung ${ }^{1, \dagger}$, Tae-Sung Kim ${ }^{2, \dagger}$, Sundan Suresh ${ }^{1}$, Sok-Young Lee ${ }^{1}$ and Gyu-Taek Cho ${ }^{1{ }^{*} *}$ \\ 1 National Agrobiodiversity Center, RDA, Suwon 441-853, Korea \\ 2 Department of Molecular Genetics, Ohio State University, Columbus, OH 43210, USA \\ $\dagger$ These authors contributed equally to this work. \\ * Author to whom correspondence should be addressed; E-Mail: gtcho@korea.kr; \\ Tel.: +82-31-290-0163; Fax: +82-31-294-6029.
}

Received: 2 May 2013; in revise form: 5 July 2013 / Accepted: 12 July 2013 /

Published: 16 July 2013

\begin{abstract}
Vetch (Vicia sativa L.) is one of the most important annual forage legumes in the World due to its multiple uses (i.e., hay, grain, silage and green manure) and high nutritional value. However, detrimental cyanoalanine toxins in its plant parts including seeds and its vulnerability to hard winter conditions are currently reducing the agronomic values of vetch varieties. Moreover, the existence in the public domain of very few genomic resources, especially molecular markers, has further hampered breeding efforts. Polymorphic simple sequence repeat markers from transcript sequences (cDNA; simple sequence repeat [SSR]) were developed for Vicia sativa subsp. sativa. We found 3,811 SSR loci from 31,504 individual sequence reads, and 300 primer pairs were designed and synthesized. In total, 65 primer pairs were found to be consistently scorable when 32 accessions were tested. The numbers of alleles ranged from 2 to 19 , frequency of major alleles per locus were $0.27-0.87$, the genotype number was $2-19$, the overall polymorphism information content $(P I C)$ values were $0.20-0.86$, and the observed and expected heterozygosity values were $0.00-0.41$ and $0.264-0.852$, respectively. These markers provide a useful tool for assessing genetic diversity, population structure, and positional cloning, facilitating vetch breeding programs.
\end{abstract}

Keywords: cDNA-SSR; genetic diversity; 454 sequencing; Vicia stiva subsp. sativa 


\section{Introduction}

Vicia sativa subsp. sativa, known as the common vetch, is one of the most commonly grown winter cover crops, or green manure, and is also used as pasture, silage, and hay [1,2]. It is cultivated with mixtures of cereal grains, providing cool-weather weed suppression and preventing fall $\mathrm{N}$ scavenging. It has been successfully applied in vineyards and orchards [1,2]. Due to its economic and ecological advantages, common vetch is now widespread through many parts of World, including the Mediterranean basin, west and central Asia, China, eastern Asia, India, and the USA [1-3].

The common vetch produces seeds that are quite similar to those of lentils in physical appearance and are highly nutritious [3-7]. However, due to the presence of cyanoalanine toxin in the seeds, which is detrimental to mono-gastric animals, including humans, the common vetch is currently tightly restricted as a feed or food source [3-6]. Moreover, its vulnerability to severe winter conditions $\left(<-10{ }^{\circ} \mathrm{C}\right)$ further reduces its true agricultural potential $[8,9]$. Thus, to address these drawbacks, it is imperative to genetically improve this legume species either through conventional breeding or biotechnology approaches. However, a severe lack of genomic resources in the public domain has hampered such efforts.

Next-generation transcriptome sequencing is an excellent solution for enriching relevant genomic resources for non-model crop species such as the common vetch, providing functional annotations as well as genetic marker information [10-12]. In particular, cDNA-SSR markers generated from this approach can facilitate marker-assisted selection for vetch improvement programs, because these may be associated with functionally annotated transcribed genes, are cost-effective, and are easily transferable to related species [10-13]. Recently, we sequenced transcriptomes of common vetch using 454 pyrosequencing technology, and found 3,811 SSR loci from 31,504 individuals. In the present study, we developed and characterized polymorphic cDNA-SSR markers based on these transcriptome sequences to further contribute to breeding and molecular genetic studies of this species.

\section{Results and Discussion}

$V$. sativa subsp. sativa transcriptome sequencing yielded about $28 \mathrm{Mb}$ and GS De Novo yielded 86,532 raw sequencing reads, based on the GS-FLX sequencer. SSRs are one of the most popular marker systems, consisting of various numbers of tandem-repeat di-, tri-, or tetra-nucleotide DNA motifs [14].

To identify SSR markers, we used the ARGOS program with default settings for $V$. sativa subsp. sativa singleton collections. In total, 3,811 potential SSR motifs were identified, with the majority being trinucleotide $(76.3 \%)$ and dinucleotide $(14.6 \%)$ repeats. There was a low rate $(9 \%)$ of all other types of SSRs (e.g., tetra-, penta-, and hexa-nucleotide motifs) and the majority of trinucleotide SSRs had the GGT/GTG//TGG motif, followed by those with the ACC/CCA/CAC motif. In addition, $\mathrm{CT} / \mathrm{TC}, \mathrm{AT} / \mathrm{TA}$, and GA/AG motifs were abundant among the dinucleotide cDNA-SSRs. The relative proportion of SSR motif types was comparable to that of other plant species [15-20]. Kaur et al. [18] reported in theory, the frequencies of di-, tri-, tetra-, penta-, and hexanucleotide repeats should progressively decrease, based on the relative probability of replication slippage events. However, trinucleotide repeat units were predominant, followed by tetra-, di-, hexa-, and pentanucleotide repeat units.

Among the identified SSR loci, we selected 100 primer pairs on the basis of same annealing temperature, only 65 primer pairs produced single dominant polymerase chain reaction (PCR) products 
that were scorable for 32 accessions (Table 1 and Figure S1), The selected 65 polymorphic primer pairs sequences that were deposited in GenBank to provide a foundation for community genomic resources for vetch breeding and biotechnology research. The number of alleles $\left(N_{A}\right)$ per locus varied widely among the markers (Table 2) and ranged from 2 to 19, with an average of 6.6 alleles. The frequency of major alleles $\left(M_{A F}\right)$ per locus was $0.27-0.87$ with an average of 0.508 . In addition, the $H_{O}$ values were $0.00-0.86$ with an average of 0.106 , and the $H_{E}$ values were $0.264-0.852$ with an average of 0.670. Lastly, polymorphic index content $(P I C)$ values were $0.20-0.91$, with an average of 0.59 Table 2. Considering the relatively high polymorphism levels, the cDNA-SSR markers developed in the present study will be useful for marker-assisted selection and population genetic studies to improve vetch varieties.

The dendrogram showed that the 32 common vetch accessions fell into five distinct clusters (Figure 1). Cluster 1 comprised accessions from South west Europe, West Asia and Central Europe each regions had 2 accessions. Cluster 2contained 3 accessions one from Eurasian, one from middle East Asia and one from North Africa. Cluster 3 contained eight accessions, five accessions from Central Europe and remaining accessions from South East Europe, north Europe and West Europe each region had one accessions. Cluster 4 contained six accessions, two accessions from South East Europe and one accession from each geographical region (Central Europe, South West Europe, Europe and North West Europe). Cluster 5 included nine accessions, two accessions from central Europe and two accessions from Europe regions, with rest of the accessions from East Asia, West Europe, South West Europe, Central and South East Europe and West General Europe, North Europe and West Europe each having one accession.

Dongi et al [21] reported cluster analysis of Trigonella foenumgraecum there was no clear clustering pattern of geographically closer accessions indicating that the association between genetic similarity and geographical distance was less significant. However, it is necessary to use more number of accessions from each geographical location to confirm the available pattern. 
Table 1. Chauracteristics of the 65 cDNA-SSR markers for common vetch (Vicia sativa subsp. sativa).

\begin{tabular}{|c|c|c|c|c|c|c|c|}
\hline Marker & Primer sequence (5'-3') & Motif & $\begin{array}{l}\text { GenBank } \\
\text { Acc. No. }\end{array}$ & Ta $\left({ }^{\circ} \mathbf{C}\right)$ & $\begin{array}{l}\text { BLAST top hit } \\
\text { Acc. No. }\end{array}$ & Description & E-value \\
\hline $\begin{array}{l}\text { GBSSR- } \\
\text { VSspS-020 }\end{array}$ & $\begin{array}{l}\text { F: CATTTGGCTGATCCTGTCA } \\
\text { R: GGCTTCATCATGAGACAAGAA }\end{array}$ & $(\mathrm{TAT}) 5$ & KF008486 & 55 & L19651.1 & $\begin{array}{l}\text { Pisum sativum chloroplast photosystem I } 24 \\
\mathrm{kDa} \text { light harvesting protein (lhca3) mRNA, } \\
\text { complete cds }\end{array}$ & $7 e-92$ \\
\hline $\begin{array}{l}\text { GBSSR- } \\
\text { VSspS-023 }\end{array}$ & $\begin{array}{l}\text { F: CCTGCATTCACAACCATTT } \\
\text { R: CGCCATCGATGTTTTGTT }\end{array}$ & $(\mathrm{CAT}) 5$ & KF008487 & 55 & None & None & None \\
\hline $\begin{array}{l}\text { GBSSR- } \\
\text { VSspS-024 }\end{array}$ & $\begin{array}{l}\text { F: ACGGTGTTAACGGTCACG } \\
\text { R: TCTCCAAACCGACACCAG }\end{array}$ & $(\mathrm{AGA}) 5$ & KF008488 & 55 & AF262939.1 & $\begin{array}{l}\text { Pisum sativum chloroplast protein import } \\
\text { component Toc } 159 \text { mRNA. complete cds }\end{array}$ & $1 e-160$ \\
\hline $\begin{array}{l}\text { GBSSR- } \\
\text { VSspS-028 }\end{array}$ & $\begin{array}{l}\text { F: TGAGCCGTTGACACAACA } \\
\text { R: GGCGATCCTCCTACTTGAA }\end{array}$ & (TGC)5 & KF008489 & 55 & None & None & None \\
\hline $\begin{array}{l}\text { GBSSR- } \\
\text { VSspS-037 }\end{array}$ & $\begin{array}{l}\text { F: GAAACAAGCTGAAGGCCC } \\
\text { R: TCAGGAAATGACCAAACCA }\end{array}$ & $(\mathrm{GAA}) 5$ & KF008490 & 55 & $\mathrm{X} 76774.1$ & P.sativum mRNA for HMG1 protein & $2 \mathrm{e}-123$ \\
\hline $\begin{array}{l}\text { GBSSR- } \\
\text { VSspS-038 }\end{array}$ & $\begin{array}{l}\text { F: CTCCCCAACTTGTTCCCT } \\
\text { R: GGGAACTTGTCGATGTGG }\end{array}$ & (TTC) 5 & KF008491 & 55 & BT143793 & $\begin{array}{l}\text { Medicago truncatula clone JCVI-FLMt-3P13 } \\
\text { unknown mRNA }\end{array}$ & $3 e-41$ \\
\hline $\begin{array}{l}\text { GBSSR- } \\
\text { VSspS-042 }\end{array}$ & $\begin{array}{l}\text { F: GGTTCGAGAGCTTTGCTG } \\
\text { R: CTGTGCCACTTGACCTCC }\end{array}$ & $(\mathrm{CGT}) 5$ & KF008492 & 55 & JF965421.1 & $\begin{array}{l}\text { Medicago sativa ethylene response factor } 11 \\
\text { (ERF11) mRNA, complete cds }\end{array}$ & $2 \mathrm{e}-37$ \\
\hline $\begin{array}{l}\text { GBSSR- } \\
\text { VSspS-057 }\end{array}$ & $\begin{array}{l}\text { F: GAGGTTTCCGGTGAGGAG } \\
\text { R: GTTCCAGCAGGTGAAGCA }\end{array}$ & $(\mathrm{GGT}) 7$ & KF008493 & 55 & XM_003548374.1 & $\begin{array}{l}\text { PREDICTED: Glycine max DEAD-box } \\
\text { ATP-dependent RNA helicase 31-like } \\
\text { (LOC100799999), mRNA }\end{array}$ & $7 e-28$ \\
\hline $\begin{array}{l}\text { GBSSR- } \\
\text { VSspS-066 }\end{array}$ & $\begin{array}{l}\text { F: AGGAGAGGCAAGGACCAG } \\
\text { R: CACGGCTATTTTCTTCTTTTTC }\end{array}$ & (GA) 10 & KF008494 & 55 & AB078603.1 & $\begin{array}{l}\text { Pisum sativum SN4TDR mRNA for } 110 \mathrm{kDa} \\
4 \mathrm{SNc}-\text { Tudor domin protein, complet cds }\end{array}$ & $1 \mathrm{e}-93$ \\
\hline $\begin{array}{l}\text { GBSSR- } \\
\text { VSspS-067 }\end{array}$ & $\begin{array}{l}\text { F: CAAACTTGTCACCACATATACAA } \\
\text { R: GGTGGTCACTAGTGGAGGTG }\end{array}$ & $(\mathrm{CCA}) 5$ & KF008495 & 55 & KC218790.1 & $\begin{array}{l}\text { Vicia faba clone NAC-VF- } 218 \text { microsatellite } \\
\text { sequence }\end{array}$ & $3 e-39$ \\
\hline $\begin{array}{l}\text { GBSSR- } \\
\text { VSspS-071 }\end{array}$ & $\begin{array}{l}\text { F: GTATTCCTCTGGTGGTGGG } \\
\text { R: CACCACCAAGACCTCCAA }\end{array}$ & (TGG)5 & KF008496 & 55 & XM 003617084.1 & $\begin{array}{l}\text { Medicago truncatula Heterogeneous nuclear } \\
\text { ribonucleoprotein DO (MTR 5g088220) } \\
\text { mRNA, complete cds }\end{array}$ & $9 e-108$ \\
\hline
\end{tabular}


Table 1. Cont.

\begin{tabular}{|c|c|c|c|c|c|c|c|}
\hline Marker & Primer sequence (5'-3') & Motif & $\begin{array}{l}\text { GenBank } \\
\text { Accession No. }\end{array}$ & $\begin{array}{l}\text { Ta } \\
\left({ }^{\circ} \mathrm{C}\right)\end{array}$ & $\begin{array}{l}\text { BLAST top hit } \\
\text { accession No. }\end{array}$ & Description & E-value \\
\hline $\begin{array}{l}\text { GBSSR- } \\
\text { VSspS-073 }\end{array}$ & $\begin{array}{l}\text { F: CCTCCCAATCCTCCATTC } \\
\text { R: CCCTAGTCCTCCAATTTCG }\end{array}$ & $(\mathrm{GTT}) 5$ & KF008497 & 55 & XM_004506417.1 & $\begin{array}{l}\text { PREDICTED: Cicer arietinum scarecrow-like } \\
\text { protein 6-like (LOC101497219), mRNA }\end{array}$ & $2 \mathrm{e}-58$ \\
\hline $\begin{array}{l}\text { GBSSR- } \\
\text { VSspS-075 }\end{array}$ & $\begin{array}{l}\text { F: TTCAGCAAGCCCATCATT } \\
\text { R: CGTCCGTCCAATCAACAA }\end{array}$ & (TTA) 6 & KF008498 & 55 & JF768700.1 & Lens culinaris microsatellite LcSSR535 sequence & $1 \mathrm{e}-71$ \\
\hline $\begin{array}{l}\text { GBSSR- } \\
\text { VSspS-076 }\end{array}$ & $\begin{array}{l}\text { F: CCTGGTCCCAGAAATGGT } \\
\text { R: AAGCCAGAGGGCATTGAT }\end{array}$ & (CTG)6 & KF008499 & 55 & XM_003602555.1 & $\begin{array}{l}\text { Medicago truncatula NAC domin protein } \\
\text { (MTR_3g096140) mRNA complete cds }\end{array}$ & $1 \mathrm{e}-136$ \\
\hline $\begin{array}{l}\text { GBSSR- } \\
\text { VSspS-079 }\end{array}$ & $\begin{array}{l}\text { F: AAAGCAAATTGTTAAAGAAAGGG } \\
\text { R: GAGGATGCTGCACATATGTAGTT }\end{array}$ & $(\mathrm{AAT}) 5$ & KF008500 & 55 & None & None & None \\
\hline $\begin{array}{l}\text { GBSSR- } \\
\text { VSspS-080 }\end{array}$ & $\begin{array}{l}\text { F: AATGCATGGATCGAGGTG } \\
\text { R: GAATCCATCGGCAACGTA }\end{array}$ & (TGG)5 & KF008501 & 55 & XM_004510745.1 & $\begin{array}{l}\text { PREDICTED: Cicer arietinum uncharacterized } \\
\text { LOC } 101512367 \text { (LOC 101512367), transcript } \\
\text { variant X3, mRNA }\end{array}$ & $6 e-110$ \\
\hline $\begin{array}{l}\text { GBSSR- } \\
\text { VSspS-088 }\end{array}$ & $\begin{array}{l}\text { F: CGAAGAGGTAAATGACGCC } \\
\text { R: AGTGACCTATATTTAGCATCGTT }\end{array}$ & (TGG)5 & KF008502 & 55 & None & None & None \\
\hline $\begin{array}{l}\text { GBSSR- } \\
\text { VSspS-090 }\end{array}$ & $\begin{array}{l}\text { F: AGACGCACCACAACAGAAA } \\
\text { R: GGGCTAGACATGGCACAA }\end{array}$ & $(\mathrm{AGC}) 6$ & KF008503 & 55 & BT149661.1 & $\begin{array}{l}\text { Medicago truncatula clone JCVI-FLMt-1507 } \\
\text { unknown mRNA }\end{array}$ & $7 e-62$ \\
\hline $\begin{array}{l}\text { GBSSR- } \\
\text { VSspS-091 }\end{array}$ & $\begin{array}{l}\text { F: CCAAACCAGCAAGAGCAG } \\
\text { R: GAGCAGCGTTGTCTCGTC }\end{array}$ & $(\mathrm{CTT}) 5$ & KF008504 & 55 & XM_003594377.1 & $\begin{array}{l}\text { Medicago trancatula Endoglucanase } \\
\text { (MTR_2g028480) mRNA complete cds }\end{array}$ & $\mathrm{e}-122$ \\
\hline $\begin{array}{l}\text { GBSSR- } \\
\text { VSspS-099 }\end{array}$ & $\begin{array}{l}\text { F: ATCCATGCCTCTTTTGCC } \\
\text { R: AGCCTCATTTCAGCAGCA }\end{array}$ & $(\mathrm{TCT}) 5$ & KF008505 & 55 & BT137674.1 & $\begin{array}{l}\text { Medicago truncatula clone JCVIMt-1708 } \\
\text { unknown mRNA }\end{array}$ & $3 e-77$ \\
\hline $\begin{array}{l}\text { GBSSR- } \\
\text { VSspS-102 }\end{array}$ & $\begin{array}{l}\text { F: TTCAACGGAGATGGATCG } \\
\text { R: CGTCTTCTTTCAGAGGCG }\end{array}$ & $(\mathrm{GTT}) 5$ & KF008506 & 55 & X59773.1 & $\begin{array}{l}\text { Pisum sativum mRNA for P protein. a part of } \\
\text { glycine cleavage complex }\end{array}$ & 0.0 \\
\hline $\begin{array}{l}\text { GBSSR- } \\
\text { VSspS-107 }\end{array}$ & $\begin{array}{l}\text { F: TGGTTTCTTTCTAAAGGGGTG } \\
\text { R: CGGCTCGATGGACAGTAG }\end{array}$ & $(\mathrm{GTT}) 5$ & KF008507 & 55 & BT146949.1 & $\begin{array}{l}\text { Medicago truncatula clone JCVI-FLMt-19I1 } \\
\text { unknown }\end{array}$ & $\mathrm{e}-127$ \\
\hline
\end{tabular}


Table 1. Cont.

\begin{tabular}{|c|c|c|c|c|c|c|c|}
\hline Marker & Primer sequence (5'-3') & Motif & $\begin{array}{l}\text { GenBank } \\
\text { Accession No. }\end{array}$ & $\begin{array}{l}\text { Ta } \\
\left({ }^{\circ} \mathrm{C}\right)\end{array}$ & $\begin{array}{l}\text { BLAST top hit } \\
\text { accession No. }\end{array}$ & Description & E-value \\
\hline $\begin{array}{l}\text { GBSSR- } \\
\text { VSspS-115 }\end{array}$ & $\begin{array}{l}\text { F: CATAAACAAGGGCAAGAAAA } \\
\text { R: GAGGAAAACATTGGTGGGA }\end{array}$ & (TGC)6 & KF008508 & 55 & XM_004501895.1 & $\begin{array}{l}\text { PREDICTED: Cicer arietinum nucleobase- ascorbate } \\
\text { transporter 6-like (LOC101504609), transcript X2, } \\
\text { mRNA }\end{array}$ & $6 e-59$ \\
\hline $\begin{array}{l}\text { GBSSR- } \\
\text { VSspS-117 }\end{array}$ & $\begin{array}{l}\text { F: CGGTGCACTAAGTGGGAA } \\
\text { R: TTAATGATGGTGGCGAGG }\end{array}$ & $(\mathrm{AGG}) 5$ & KF008509 & 55 & BT135350.1 & $\begin{array}{l}\text { Medicago truncatula clone JCVI-FLMt-9H4 unknown } \\
\text { mRNA }\end{array}$ & $2 \mathrm{e}-114$ \\
\hline $\begin{array}{l}\text { GBSSR- } \\
\text { VSspS-118 }\end{array}$ & $\begin{array}{l}\text { F: GCATTTCCCTTGGTCTCC } \\
\text { R: CAGAAAGAGCAACCGTGC }\end{array}$ & (TGG)5 & KF008510 & 55 & XM_004514512.1 & $\begin{array}{l}\text { PREDICTED: Cicer arietinum N-alpha- } \\
\text { acetyltransferase 10-like (LOC101504041), mRNA }\end{array}$ & $3 e-68$ \\
\hline $\begin{array}{l}\text { GBSSR- } \\
\text { VSspS-119 }\end{array}$ & $\begin{array}{l}\text { F: CACCACCAAGACCTCCAA } \\
\text { R: CCATCATCATCACCAGCC }\end{array}$ & $(\mathrm{ACC}) 5$ & KF008511 & 55 & XM_003617084.1 & $\begin{array}{l}\text { Medicago truncatula Heterogeneous nuclear } \\
\text { ribonucleoprotein D0 (MTR_5g088220), complete cds }\end{array}$ & $9 e-98$ \\
\hline $\begin{array}{l}\text { GBSSR- } \\
\text { VSspS-125 }\end{array}$ & $\begin{array}{l}\text { F: GGCCGGTATTCGTCAACT } \\
\text { R: CCCCGTATTTTCTCGGTC }\end{array}$ & (TGG)5 & KF008512 & 55 & XM_004510745.1 & $\begin{array}{l}\text { PREDICTED: Cicer arietinum uncharacterized LOC } \\
101512367 \text { (LOC101512367), transcript variant X3, } \\
\text { mRNA }\end{array}$ & $2 \mathrm{e}-134$ \\
\hline $\begin{array}{l}\text { GBSSR- } \\
\text { VSspS-126 }\end{array}$ & $\begin{array}{l}\text { F: TGGCGCTTATCGCTATGT } \\
\text { R: TCCACTCATTCCACTCGT }\end{array}$ & (TG)7 & KF008513 & 55 & XM_003623363.1 & $\begin{array}{l}\text { Medicago truncatula Patellin-6 (TR_7G070480) } \\
\text { mRNA, complete cds }\end{array}$ & $5 e-81$ \\
\hline $\begin{array}{l}\text { GBSSR- } \\
\text { VSspS-129 }\end{array}$ & $\begin{array}{l}\text { F: AGGAGAGGCAAGGACCAG } \\
\text { R: CTTTTTCTCTAACTCATTCATGTC }\end{array}$ & (GA) 10 & KF008514 & 55 & AB0786031 & $\begin{array}{l}\text { Pisum sativum SN4TDR mRNA for } 110 \mathrm{kDa} 4 \mathrm{SNc}- \\
\text { Tudor domain protein, complete cds }\end{array}$ & $8 \mathrm{e}-117$ \\
\hline $\begin{array}{l}\text { GBSSR- } \\
\text { VSspS-135 }\end{array}$ & $\begin{array}{l}\text { F: TGGTGGAGATTTGTTGGG } \\
\text { R: CTTCATCTTCCCACACCG }\end{array}$ & (TGG)5 & KF008515 & 55 & BT136030.1 & $\begin{array}{l}\text { Medicago truncatula clone JCVI-FLMt-16L14unknown } \\
\text { mRNA }\end{array}$ & $4 \mathrm{e}-40$ \\
\hline $\begin{array}{l}\text { GBSSR- } \\
\text { VSspS-138 }\end{array}$ & $\begin{array}{l}\text { F: CGGAGTTCACATAAAACATACTAC } \\
\text { R: TGGGAGTGTTGAGATGGG }\end{array}$ & (TTA) 7 & KF008516 & 55 & AB176563.1 & $\begin{array}{l}\text { Vicia faba MET mRNA for type } 2 \text { metallothionein, } \\
\text { complete cds }\end{array}$ & $6 e-98$ \\
\hline $\begin{array}{l}\text { GBSSR- } \\
\text { VSspS-140 }\end{array}$ & $\begin{array}{l}\text { F: TTGCTTTGATGTTTGGAGC } \\
\text { R: CCCTAAATTCCCAACCCA }\end{array}$ & $(\mathrm{GGT}) 7$ & KF008517 & 55 & XM_003613856.1 & $\begin{array}{l}\text { Medicago truncatula Cysteine-rich receptor-like protein } \\
\text { kinase (MTR_5g042440) mRNA, complete cds }\end{array}$ & $3 e-86$ \\
\hline
\end{tabular}


Table 1. Cont

\begin{tabular}{|c|c|c|c|c|c|c|c|}
\hline Marker & Primer sequence (5'-3') & Motif & $\begin{array}{l}\text { GenBank } \\
\text { Accession No. }\end{array}$ & $\begin{array}{l}\text { Ta } \\
\left({ }^{\circ} \mathrm{C}\right)\end{array}$ & $\begin{array}{l}\text { BLAST top hit } \\
\text { accession No. }\end{array}$ & Description & E-value \\
\hline $\begin{array}{l}\text { GBSSR- } \\
\text { VSspS-156 }\end{array}$ & $\begin{array}{l}\text { F: GGCCAATTTAGCGAGCTT } \\
\text { R: CACTATCATCAACCTCTAACGGA }\end{array}$ & (GTG)5 & KF008519 & 55 & BT134176.1 & $\begin{array}{l}\text { Medicago truncatula clone JCVI-FLMt-15D24 } \\
\text { unknown mRNA }\end{array}$ & $2 \mathrm{e}-118$ \\
\hline $\begin{array}{l}\text { GBSSR- } \\
\text { VSspS-158 }\end{array}$ & $\begin{array}{l}\text { F: TGAGCTTATTGCCAGTGGA } \\
\text { R: CCATGTCATCATCGGATTC }\end{array}$ & (TGG)5 & KF008520 & 55 & KC218603.1 & Vicia faba clone NAC-VF-31 microsatellite sequence & $7 \mathrm{e}-124$ \\
\hline $\begin{array}{l}\text { GBSSR- } \\
\text { VSspS-162 }\end{array}$ & $\begin{array}{l}\text { F: GAGACAGTGGAAGTATCGGC } \\
\text { R: CACAGCAAATGCATCGGT }\end{array}$ & $(\mathrm{AAG}) 6$ & KF008521 & 55 & BT146412.1 & $\begin{array}{l}\text { Medicago truncatula clone JCVI-FLMt-21014 } \\
\text { unknown mRNA }\end{array}$ & $1 e-75$ \\
\hline $\begin{array}{l}\text { GBSSR- } \\
\text { VSspS-166 }\end{array}$ & $\begin{array}{l}\text { F: GTGGCCATGATCCATTTG } \\
\text { R: TTCCTCGAGAGGGAAAGC }\end{array}$ & (TGG)5 & KF008522 & 55 & XM_003605932.1 & $\begin{array}{l}\text { Medicago truncatula DnaJ (MTR_4g50420) mRNA } \\
\text { complete cds }\end{array}$ & 0.0 \\
\hline $\begin{array}{l}\text { GBSSR- } \\
\text { VSspS-172 }\end{array}$ & $\begin{array}{l}\text { F: GCTTTGGAAGAGCCCAAT } \\
\text { R: TCCAGGATTGTAACCCCC }\end{array}$ & $(\mathrm{TGG}) 5$ & KF008523 & 55 & XM_003617084.1 & $\begin{array}{l}\text { Medicago truncatula Heterogeneous nuclear } \\
\text { ribonucleoprotein D0 (MTR_5g088220) mRNA, } \\
\text { complete cds }\end{array}$ & $5 e-90$ \\
\hline $\begin{array}{l}\text { GBSSR- } \\
\text { VSspS-173 }\end{array}$ & $\begin{array}{l}\text { F: GGGCACGGTGGTCACTA } \\
\text { R: TGACTACCACCACCTCCG }\end{array}$ & $(\mathrm{TGG}) 5$ & KF008524 & 55 & AJ831469.1 & $\begin{array}{l}\text { Pisum sativum mRNA for putative glycine rich } \\
\text { protein precursor (grp1 gene) }\end{array}$ & $2 \mathrm{e}-13$ \\
\hline $\begin{array}{l}\text { GBSSR- } \\
\text { VSspS-179 }\end{array}$ & $\begin{array}{l}\text { F: AGCTATGCGAGAGGCTCC } \\
\text { R: CTGTGGGAAGGCACATCT }\end{array}$ & (TGA)6 & KF008525 & 55 & None & None & None \\
\hline $\begin{array}{l}\text { GBSSR- } \\
\text { VSspS-181 }\end{array}$ & $\begin{array}{l}\text { F: CACTGTGACTCAGTTTCGTTG } \\
\text { R: CGATTTTGAACCCTAACCG }\end{array}$ & (TTC)5 & KF008526 & 55 & None & None & None \\
\hline $\begin{array}{l}\text { GBSSR- } \\
\text { VSspS-182 }\end{array}$ & $\begin{array}{l}\text { F: GCGTTGTGGCGTATTTCT } \\
\text { R: TGGAGGAAAGGAAACTACTCA }\end{array}$ & $(\mathrm{GCA}) 6$ & KF008527 & 55 & AB676029.1 & Lathyrus japonicas DNA, 61 locus, haplotype: D & $4 e-62$ \\
\hline $\begin{array}{l}\text { GBSSR- } \\
\text { VSspS-185 }\end{array}$ & $\begin{array}{l}\text { F: CTCCTCAATTTTCCCCCA } \\
\text { R: TTTGGTGCGATTGTTTCC } \\
\end{array}$ & $(\mathrm{CAT}) 5$ & KF008528 & 55 & Ap009676.1 & $\begin{array}{l}\text { Lotus japonicas genomic DNA, clone: LjT30I08, } \\
\text { TM0492 }\end{array}$ & $4 \mathrm{e}-26$ \\
\hline $\begin{array}{l}\text { GBSSR- } \\
\text { VSspS-187 }\end{array}$ & $\begin{array}{l}\text { F: CCAGGTTGCTTTCCTTACTTT } \\
\text { R: TTAGCCCTCAAAGCCTCC }\end{array}$ & (ATC) 5 & KF008529 & 55 & X54359.1 & P.sativum mRNA of cDNA clone $26 \mathrm{~g}$ & $2 \mathrm{e}-154$ \\
\hline $\begin{array}{l}\text { GBSSR- } \\
\text { VSspS-192 }\end{array}$ & $\begin{array}{l}\text { F: AGGGTCTTCCTTCCCACA } \\
\text { R: TATGGTGACACGTTCGCA }\end{array}$ & $(\mathrm{ATC}) 5$ & KF008530 & 55 & XM_004505980.1 & $\begin{array}{l}\text { PREDICTED: Cicer arietinum uncharacterized } \\
\text { LOC101500025 (LOC101500025), transcript variant } \\
\text { X2, mRNA }\end{array}$ & $1 \mathrm{e}-100$ \\
\hline
\end{tabular}


Table 1. Cont

\begin{tabular}{|c|c|c|c|c|c|c|c|}
\hline Marker & Primer sequence (5'-3') & Motif & $\begin{array}{l}\text { GenBank } \\
\text { Accession No. }\end{array}$ & $\begin{array}{l}\text { Ta } \\
\left({ }^{\circ} \mathbf{C}\right) \\
\end{array}$ & $\begin{array}{l}\text { BLAST top hit } \\
\text { accession No. }\end{array}$ & Description & E-value \\
\hline $\begin{array}{l}\text { GBSSR- } \\
\text { VSspS-203 }\end{array}$ & $\begin{array}{l}\text { F: TCCATCTGGTTGGTGGTG } \\
\text { R: GAAAGCCAATTTTTCAGCAA }\end{array}$ & $(\mathrm{GTT}) 7$ & KF008531 & 55 & BT147294.1 & $\begin{array}{l}\text { Medicago truncatula clone JCVI-FLMt-15A20 } \\
\text { unknown mRNA }\end{array}$ & $2 \mathrm{e}-50$ \\
\hline $\begin{array}{l}\text { GBSSR- } \\
\text { VSspS-217 }\end{array}$ & $\begin{array}{l}\text { F: CCATCGCCACCACCA } \\
\text { R: TCCCGGAACAAAAATCAA }\end{array}$ & $(\mathrm{AAC}) 7$ & KF008532 & 55 & EF447278.1 & $\begin{array}{l}\text { Pisum sativum cultivar Finale SYM8 (SYM8) gene, } \\
\text { partial cds }\end{array}$ & $5 e-58$ \\
\hline $\begin{array}{l}\text { GBSSR- } \\
\text { VSspS-245 }\end{array}$ & $\begin{array}{l}\text { F: CAATAGGGGGACCCTTCA } \\
\text { R: GCTGCAAGCTGCTACCAT }\end{array}$ & $(\mathrm{GGA}) 5$ & KF008533 & 55 & HQ439603.1 & $\begin{array}{l}\text { Phalaenopsis hybrid cultivar candidate developmental } \\
\text { transcription factor TCP1 mRNA, partial cds }\end{array}$ & $5 e-04$ \\
\hline $\begin{array}{l}\text { GBSSR- } \\
\text { VSspS-247 }\end{array}$ & $\begin{array}{l}\text { F: GGTTCAATACGATCCATAGAATA } \\
\text { R: TGATCGCCAATTCTGGAC }\end{array}$ & $(\mathrm{CAC}) 6$ & KF008534 & 55 & KC294548.1 & $\begin{array}{l}\text { Aeschynomene ciliate voucher IRRI } 13078 \text { cyclophilin } \\
1 \text { (CYP1) gene, complete cds }\end{array}$ & $3 e-46$ \\
\hline $\begin{array}{l}\text { GBSSR- } \\
\text { VSspS-249 }\end{array}$ & $\begin{array}{l}\text { F: AAAACATGGTTGAGTGTTTTTG } \\
\text { R: TAACCCTCTCGGTTTCGG }\end{array}$ & (ATA)5 & KF008535 & 55 & KC218749.1 & Vicia faba clone NAC-VF-177 microsatellite sequence & $2 \mathrm{e}-55$ \\
\hline $\begin{array}{l}\text { GBSSR- } \\
\text { VSspS-251 }\end{array}$ & $\begin{array}{l}\text { F: TGGTGGACGTCACTATGGA } \\
\text { R: CATGGTGCTTCCGACAAT }\end{array}$ & (TGG)5 & KF008536* & 55 & KC218790.1 & Vicia faba clone NAC-VF-218 microsatellite sequence & $5 e-30$ \\
\hline $\begin{array}{l}\text { GBSSR- } \\
\text { VSspS-252 }\end{array}$ & $\begin{array}{l}\text { F: CATGGTGCTTCCGACAAT } \\
\text { R: TCGAAATCAGGACTTACCACA }\end{array}$ & $(\mathrm{CCA}) 5$ & KF008536 * & 55 & KC218790.1 & Vicia faba clone NAC-VF-218 microsatellite sequence & $5 e-30$ \\
\hline $\begin{array}{l}\text { GBSSR- } \\
\text { VSspS-262 }\end{array}$ & $\begin{array}{l}\text { F: ATTGGGCCCTCTTTTTGA } \\
\text { R: GGGGGTAGAAAAGTTGCG }\end{array}$ & $(\mathrm{AT}) 7$ & KF008537 & 55 & XM_004500781.1 & $\begin{array}{l}\text { PREDICTED: Cicer arietinum dolichyl- } \\
\text { diphoshooligosaccharide-protein glycosyltransferase } \\
\text { subunit 1A-like (LOC101502563), mRNA }\end{array}$ & $1 e-37$ \\
\hline $\begin{array}{l}\text { GBSSR- } \\
\text { VSspS-268 }\end{array}$ & $\begin{array}{l}\text { F: AAATTTGTCTGACGAAAAACG } \\
\text { R: TGCTTGAGAGTGCCATCA }\end{array}$ & $(\mathrm{TAC}) 5$ & KF008538 & 55 & BT144509.1 & $\begin{array}{l}\text { Medicago truncatula clone JCVI-FLMt-13M6 unknown } \\
\text { mRNA }\end{array}$ & $2 \mathrm{e}-47$ \\
\hline $\begin{array}{l}\text { GBSSR- } \\
\text { VSspS-269 }\end{array}$ & $\begin{array}{l}\text { F: TTCCATTTATCCTCCTATCCTCT } \\
\text { R: CTTGAATGCGAAACGAGG }\end{array}$ & $(\mathrm{CGC}) 5$ & KF008539 & 55 & XM_004507695.1 & $\begin{array}{l}\text { PREDICTED: Cicer arietinum eukaryotic translation } \\
\text { initiation factorisoform 4G-1-like (LOC101492356), } \\
\text { mRNA }\end{array}$ & $1 e-56$ \\
\hline $\begin{array}{l}\text { GBSSR- } \\
\text { VSspS-284 }\end{array}$ & $\begin{array}{l}\text { F: TGGAAGGAAATGGCAGTG } \\
\text { R: ATCCGTTTCGGATTGGTT }\end{array}$ & $(\mathrm{GCA}) 5$ & KF008540 & 55 & JX539287.1 & $\begin{array}{l}\text { Vigna radiata cultivar MCV-1 clone GGSSR_911 } \\
\text { microsatellit sequence }\end{array}$ & $2 \mathrm{e}-120$ \\
\hline
\end{tabular}


Table 1. Cont

\begin{tabular}{|c|c|c|c|c|c|c|c|}
\hline Marker & Primer sequence (5'-3') & Motif & $\begin{array}{l}\text { GenBank } \\
\text { Accession No. }\end{array}$ & $\begin{array}{l}\text { Ta } \\
\left({ }^{\circ} \mathrm{C}\right)\end{array}$ & $\begin{array}{l}\text { BLAST top hit } \\
\text { accession No. }\end{array}$ & Description & E-value \\
\hline $\begin{array}{l}\text { GBSSR- } \\
\text { VSspS-291 }\end{array}$ & $\begin{array}{l}\text { F: CCCAACCGAACCACTTATT } \\
\text { R: TAATAGCTCCGGCCCAGT }\end{array}$ & (CTA) 5 & KF008541 & 55 & None & None & None \\
\hline $\begin{array}{l}\text { GBSSR- } \\
\text { VSspS-301 }\end{array}$ & $\begin{array}{l}\text { F: AACCAAACAACAATGGGTT } \\
\text { R: TCAACCGGTGAAAGATGG }\end{array}$ & $(\mathrm{CAA}) 5$ & KF008542 & 55 & JN849865.1 & $\begin{array}{l}\text { Medicago falcata voucher PI494662A caffeic acid-O- } \\
\text { methyltransferase (COMT) gene.exon } 1 \text { and partial cds }\end{array}$ & $2 \mathrm{e}-128$ \\
\hline $\begin{array}{l}\text { GBSSR- } \\
\text { VSspS-304 }\end{array}$ & $\begin{array}{l}\text { F: CCGTTCTACGCAATTCTCC } \\
\text { R: CGACCAAGAACACCAGGA }\end{array}$ & (TTC)5 & KF008543 & 55 & XM_003593951.1 & $\begin{array}{l}\text { Medicago truncatula hypothetical protein } \\
\text { (MTR_2g020190) mRNA,complete cds }\end{array}$ & $1 \mathrm{e}-55$ \\
\hline $\begin{array}{l}\text { GBSSR- } \\
\text { VSspS-305 }\end{array}$ & $\begin{array}{l}\text { F: CATGAAAGAGTTTTGCACCTT } \\
\text { R: CCGACGACGAGATTGAGA }\end{array}$ & $(\mathrm{GCA}) 5$ & KF008544 & 55 & None & None & None \\
\hline $\begin{array}{l}\text { GBSSR- } \\
\text { VSspS-308 }\end{array}$ & $\begin{array}{l}\text { F: TGAGAGCATAGACAGCAAACA } \\
\text { R: TGGATTTGGTCGCATAGC }\end{array}$ & $(\mathrm{AAC}) 5$ & KF008545 & 55 & None & None & None \\
\hline $\begin{array}{l}\text { GBSSR- } \\
\text { VSspS-309 }\end{array}$ & $\begin{array}{l}\text { F: TCTTCAAAAGAGTACAAAAGGGA } \\
\text { R: GAATTGGACACCTTGGCA }\end{array}$ & $(\mathrm{AAT}) 5$ & KF008546 & 55 & ВT137399.1 & $\begin{array}{l}\text { Medicago truncatula clone JCVI-FlMt-19L22 unknown } \\
\text { mRNA }\end{array}$ & $5 \mathrm{e}-124$ \\
\hline $\begin{array}{l}\text { GBSSR- } \\
\text { VSspS-310 }\end{array}$ & $\begin{array}{l}\text { F: GGGTGCCCTAGCATTTGT } \\
\text { R: ATCTCCGGCGTCAGTTTC }\end{array}$ & (CTC)6 & KF008547 & 55 & M69105.1 & $\begin{array}{l}\text { Pisum sativum outer membrane protein (OM14) } \\
\text { mRNA, complete cds }\end{array}$ & $9 e-96$ \\
\hline $\begin{array}{l}\text { GBSSR- } \\
\text { VSspS-311 }\end{array}$ & $\begin{array}{l}\text { F: TTGAGGCGGTGTTGGTAG } \\
\text { R: ATGTCATGGCCAACTGCT }\end{array}$ & $(\mathrm{GGA}) 6$ & KF008548 & 55 & None & None & None \\
\hline $\begin{array}{l}\text { GBSSR- } \\
\text { VSspS-313 }\end{array}$ & $\begin{array}{l}\text { F: GAACAATGCAGCCTGGAA } \\
\text { R: GCTGCAATCGCATTCTCT }\end{array}$ & (TTG)5 & KF008549 & 55 & XM_003613196.1 & $\begin{array}{l}\text { Medicago truncatula U-box domin containing protein } \\
\text { (MTR_5g034440) mRNA, complete cds }\end{array}$ & $2 \mathrm{e}-112$ \\
\hline
\end{tabular}

TA, annealing temperature. * same sequence two primers identified. 
Table 2. Diversity statistics from initial primer screening in 32 accessions of common vetch (Vicia sativa subsp. sativa).

\begin{tabular}{|c|c|c|c|c|c|}
\hline Marker & $N_{A}$ & $M_{A F}$ & $\boldsymbol{H}_{\boldsymbol{O}}$ & $\boldsymbol{H}_{E}$ & $P I C$ \\
\hline GBSSR-VSspS-020 & 4 & 0.63 & 0.00 & 0.51 & 0.43 \\
\hline GBSSR-VSspS-023 & 7 & 0.34 & 0.00 & 0.75 & 0.71 \\
\hline GBSSR-VSspS-024 & 7 & 0.47 & 0.00 & 0.71 & 0.67 \\
\hline GBSSR-VSspS-028 & 4 & 0.38 & 0.00 & 0.73 & 0.68 \\
\hline GBSSR-VSspS-037 & 4 & 0.38 & 0.00 & 0.71 & 0.65 \\
\hline GBSSR-VSspS-038 & 7 & 0.28 & 0.00 & 0.79 & 0.76 \\
\hline GBSSR-VSspS-042 & 2 & 0.84 & 0.00 & 0.26 & 0.23 \\
\hline GBSSR-VSspS-057 & 8 & 0.36 & 0.03 & 0.77 & 0.74 \\
\hline GBSSR-VSspS-066 & 7 & 0.25 & 0.00 & 0.83 & 0.81 \\
\hline GBSSR-VSspS-067 & 6 & 0.50 & 0.00 & 0.67 & 0.62 \\
\hline GBSSR-VSspS-071 & 3 & 0.50 & 0.00 & 0.62 & 0.54 \\
\hline GBSSR-VSspS-073 & 8 & 0.31 & 0.00 & 0.81 & 0.79 \\
\hline GBSSR-VSspS-075 & 6 & 0.38 & 0.03 & 0.76 & 0.72 \\
\hline GBSSR-VSspS-076 & 3 & 0.56 & 0.00 & 0.57 & 0.50 \\
\hline GBSSR-VSspS-079 & 6 & 0.55 & 0.00 & 0.64 & 0.60 \\
\hline GBSSR-VSspS-080 & 3 & 0.50 & 0.00 & 0.59 & 0.51 \\
\hline GBSSR-VSspS-088 & 5 & 0.38 & 0.00 & 0.74 & 0.70 \\
\hline GBSSR-VSspS-090 & 7 & 0.41 & 0.00 & 0.75 & 0.71 \\
\hline GBSSR-VSspS-091 & 4 & 0.47 & 0.00 & 0.68 & 0.64 \\
\hline GBSSR-VSspS-099 & 4 & 0.56 & 0.00 & 0.60 & 0.54 \\
\hline GBSSR-VSspS-102 & 8 & 0.44 & 0.03 & 0.75 & 0.73 \\
\hline GBSSR-VSspS-107 & 3 & 0.66 & 0.00 & 0.51 & 0.45 \\
\hline GBSSR-VSspS-115 & 4 & 0.48 & 0.03 & 0.54 & 0.44 \\
\hline GBSSR-VSspS-117 & 6 & 0.41 & 0.00 & 0.71 & 0.67 \\
\hline GBSSR-VSspS-118 & 3 & 0.53 & 0.00 & 0.61 & 0.54 \\
\hline GBSSR-VSspS-119 & 2 & 0.65 & 0.00 & 0.46 & 0.35 \\
\hline GBSSR-VSspS-125 & 3 & 0.41 & 0.00 & 0.63 & 0.56 \\
\hline GBSSR-VSspS-126 & 7 & 0.25 & 0.00 & 0.82 & 0.79 \\
\hline GBSSR-VSspS-129 & 7 & 0.25 & 0.00 & 0.81 & 0.79 \\
\hline GBSSR-VSspS-135 & 5 & 0.50 & 0.00 & 0.62 & 0.55 \\
\hline GBSSR-VSspS-138 & 11 & 0.32 & 0.06 & 0.83 & 0.81 \\
\hline GBSSR-VSspS-140 & 8 & 0.22 & 0.03 & 0.81 & 0.79 \\
\hline GBSSR-VSspS-151 & 5 & 0.67 & 0.03 & 0.52 & 0.48 \\
\hline GBSSR-VSspS-156 & 5 & 0.66 & 0.00 & 0.53 & 0.50 \\
\hline GBSSR-VSspS-158 & 5 & 0.59 & 0.00 & 0.57 & 0.52 \\
\hline
\end{tabular}


Table 2. Cont.

\begin{tabular}{|c|c|c|c|c|c|}
\hline Marker & $N_{A}$ & $M_{A F}$ & $\boldsymbol{H}_{O}$ & $\boldsymbol{H}_{E}$ & PIC \\
\hline GBSSR-VSspS-162 & 6 & 0.53 & 0.00 & 0.66 & 0.63 \\
\hline GBSSR-VSspS-166 & 6 & 0.38 & 0.00 & 0.74 & 0.70 \\
\hline GBSSR-VSspS-172 & 3 & 0.41 & 0.00 & 0.65 & 0.57 \\
\hline GBSSR-VSspS-173 & 4 & 0.58 & 0.00 & 0.58 & 0.53 \\
\hline GBSSR-VSspS-179 & 8 & 0.56 & 0.03 & 0.64 & 0.61 \\
\hline GBSSR-VSspS-181 & 11 & 0.28 & 0.00 & 0.85 & 0.84 \\
\hline GBSSR-VSspS-182 & 7 & 0.38 & 0.00 & 0.76 & 0.73 \\
\hline GBSSR-VSspS-185 & 10 & 0.31 & 0.03 & 0.82 & 0.80 \\
\hline GBSSR-VSspS-187 & 5 & 0.44 & 0.00 & 0.69 & 0.64 \\
\hline GBSSR-VSspS-192 & 3 & 0.63 & 0.00 & 0.51 & 0.43 \\
\hline GBSSR-VSspS-203 & 6 & 0.34 & 0.00 & 0.76 & 0.72 \\
\hline GBSSR-VSspS-217 & 11 & 0.28 & 0.00 & 0.83 & 0.81 \\
\hline GBSSR-VSspS-245 & 7 & 0.28 & 0.00 & 0.81 & 0.78 \\
\hline GBSSR-VSspS-247 & 6 & 0.69 & 0.03 & 0.49 & 0.46 \\
\hline GBSSR-VSspS-249 & 6 & 0.56 & 0.00 & 0.64 & 0.61 \\
\hline GBSSR-VSspS-251 & 5 & 0.45 & 0.00 & 0.70 & 0.66 \\
\hline GBSSR-VSspS-252 & 3 & 0.50 & 0.00 & 0.61 & 0.53 \\
\hline GBSSR-VSspS-262 & 9 & 0.47 & 0.00 & 0.72 & 0.70 \\
\hline GBSSR-VSspS-268 & 3 & 0.47 & 0.00 & 0.64 & 0.57 \\
\hline GBSSR-VSspS-269 & 4 & 0.50 & 0.00 & 0.58 & 0.49 \\
\hline GBSSR-VSspS-284 & 6 & 0.44 & 0.00 & 0.66 & 0.60 \\
\hline GBSSR-VSspS-291 & 5 & 0.53 & 0.00 & 0.64 & 0.59 \\
\hline GBSSR-VSspS-301 & 3 & 0.53 & 0.00 & 0.58 & 0.50 \\
\hline GBSSR-VSspS-304 & 7 & 0.46 & 0.00 & 0.68 & 0.63 \\
\hline GBSSR-VSspS-305 & 3 & 0.69 & 0.00 & 0.48 & 0.43 \\
\hline GBSSR-VSspS-308 & 6 & 0.54 & 0.00 & 0.66 & 0.63 \\
\hline GBSSR-VSspS-309 & 8 & 0.34 & 0.00 & 0.78 & 0.75 \\
\hline GBSSR-VSspS-310 & 8 & 0.38 & 0.03 & 0.75 & 0.71 \\
\hline GBSSR-VSspS-311 & 7 & 0.44 & 0.00 & 0.73 & 0.70 \\
\hline GBSSR-VSspS-313 & 5 & 0.44 & 0.00 & 0.71 & 0.67 \\
\hline Mean & 5.7 & 0.460 & 0.006 & 0.670 & 0.624 \\
\hline
\end{tabular}

$N A$, number of alleles; $M A F$, major allele frequency; $H O$, observed heterozygosity; $H E$, expected heterozygosity; PIC, polymorphic information content. 
Figure 1. Dendrogram generated using UPGMA cluster analysis based on genetic diversity of 32 common vetch (Vicia sativa subsp. sativa) accessions.

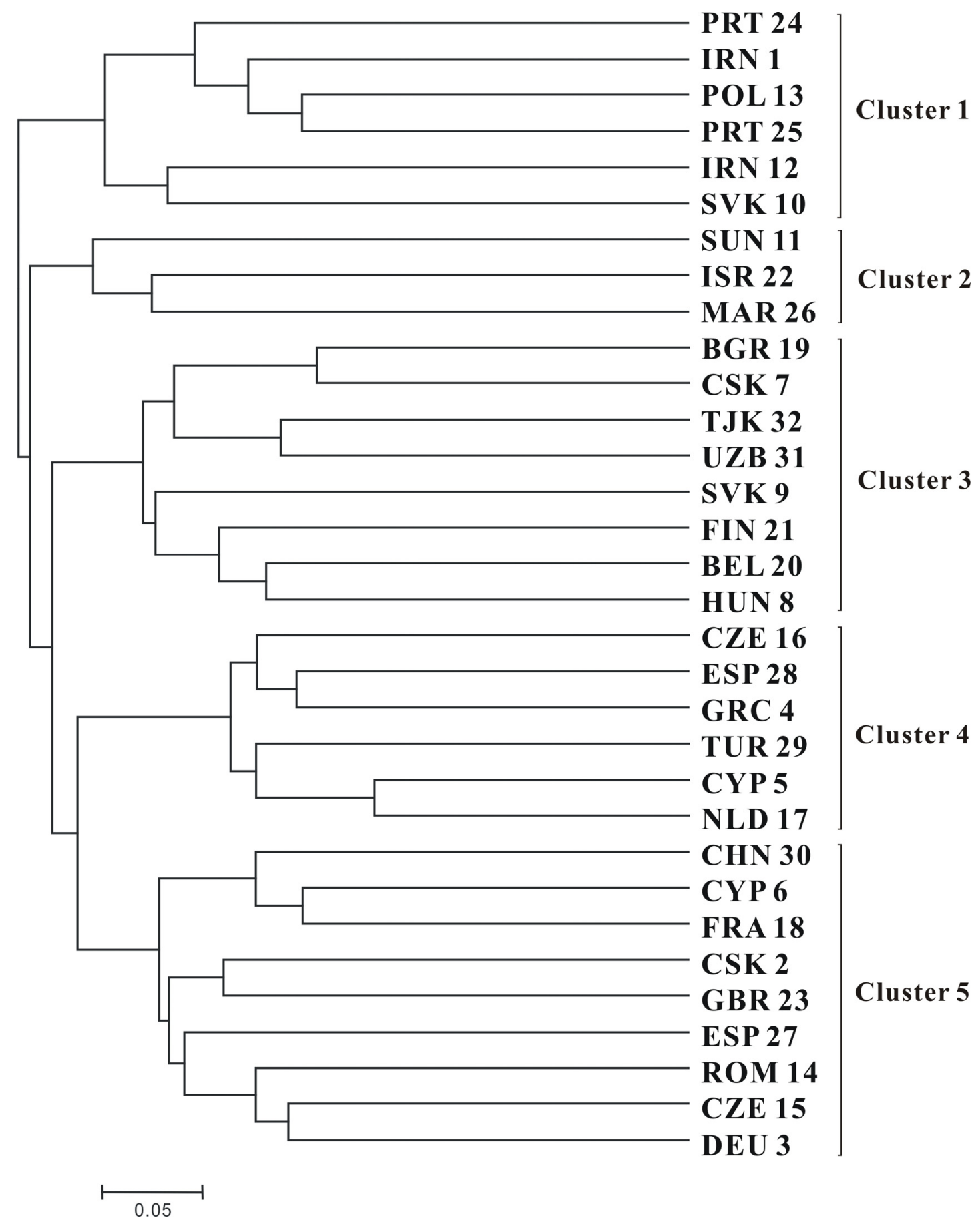

\section{Experimental}

\subsection{Plant Material}

Vicia sativa sativa seeds were selected from the National Agrobiodiversity Center, Rural Development Administration, Suwon, Korea (Table 3). Seedlings were germinated and grown in a glasshouse. The leaves of young seedlings were used to extract the mRNA required to synthesize the cDNA library and for 454 sequencing. 
Table 3. List of common vetch (Vicia sativa subsp. sativa) accessions.

\begin{tabular}{|c|c|c|c|c|}
\hline No. & Temp. ID & USDA-ARS No. & Country of origin & Geographical region of origin \\
\hline 1 & K193581 & PI 226487 & Iran & West Asia \\
\hline 2 & K193582 & PI 284058 & Czech Republic & Central Europe \\
\hline 3 & K193583 & PI 284068 & Germany & Western Central Europe \\
\hline 4 & K193584 & PI 284078 & Greece & South East Europe \\
\hline 5 & K193585 & PI 284402 & Cyprus & Europe \\
\hline 6 & K193586 & PI 284409 & Cyprus & Europe \\
\hline 7 & K193587 & PI 284470 & Czech Republic & Central Europe \\
\hline 8 & K193588 & PI 284471 & Hungary & Central Europe \\
\hline 9 & K193589 & PI 308111 & Slovakia & Central Europe \\
\hline 10 & K193590 & PI 308118 & Slovakia & Central Europe \\
\hline 11 & K193591 & PI 325513 & Soviet Union & Eurasian \\
\hline 12 & K193592 & PI 381065 & Iran & West Asia \\
\hline 13 & K193593 & PI 393870 & Poland & Central Europe \\
\hline 14 & K193594 & PI 393871 & Romania & Central and South East Europe \\
\hline 15 & K193595 & PI 393872 & Czech Republic & Central Europe \\
\hline 16 & K193596 & PI 393873 & Czech Republic & Central Europe \\
\hline 17 & K193597 & PI 393877 & Netherlands & North West Europe \\
\hline 18 & K193598 & PI 393878 & France & West Europe \\
\hline 19 & K193599 & PI 393891 & Bulgaria & South East Europe \\
\hline 20 & K193600 & PI 393904 & Belgium & West Europe \\
\hline 21 & K193601 & PI 393907 & Finland & North Europe \\
\hline 22 & K193602 & PI 393909 & Israel & Middle East Asia \\
\hline 23 & K193603 & PI 393910 & United Kingdom & Europe \\
\hline 24 & K193604 & PI 393912 & Portugal & South West Europe \\
\hline 25 & K193605 & PI 493307 & Portugal & South West Europe \\
\hline 26 & K193606 & PI 517191 & Morocco & North Africa \\
\hline 27 & K193607 & PI 533741 & Spain & South West Europe \\
\hline 28 & K193608 & PI 533743 & Spain & South West Europe \\
\hline 29 & K193609 & PI 557498 & Turkey & South East Europe \\
\hline 30 & K193610 & PI 577751 & China & East Asia \\
\hline 31 & K193611 & PI 628288 & Uzbekistan & Central Europe \\
\hline 32 & K193612 & PI 664293 & Tajikistan & Central Europe \\
\hline
\end{tabular}

(Temp ID), Korean GeneBank ID; (ARS No.), USDA-ARS Number.

\section{2. cDNA Preparation}

Total RNA was extracted from Vicia sativa subsp. sativa leaves that were frozen in liquid nitrogen, ground into a powder, and then extracted using an RNeasy Plant Mini kit (Qiagen, Valencia, CA, USA) following the manufacturer's instructions. The integrity of total RNA was determined using a BIOSPEC-NANO spectrophotometer (Shimadzu, Kyoto, Japan) and agarose gel electrophoresis. mRNA was purified using the PolyATract mRNA Isolation System IV (Promega, Madison, WI, USA), and the purified products were used to synthesize full-length cDNAs using a ZAP-cDNA Synthesis kit (Stratagene, Santa Clara, CA, USA). Finally, cDNA was fragmented by nebulization for library construction. 


\subsection{Library Preparation}

Approximately $1 \mu \mathrm{g}$ cDNA was used to generate a DNA library to use with the Genome Sequencer GS-FLX Titanium System (Roche, 454 Life Science, Branford, CT, USA). The cDNA fragment ends were polished (blunted), and two short adapters were ligated to both ends according to standard procedures described previously. The adapters, along with the sequencing key, a short sequence of four nucleotides used by the system's software for base calling, provided priming of the sequences for both the amplification and sequencing of the sample library fragments. Following the repair of any nicks in the double-stranded library, the unbound strand of each fragment was released (with 5-Adaptor A). Finally, the quality of this single-stranded template DNA library was assessed using a 2100 BioAnalyzer (Agilent, Waldbronn, Germany). The library was quantified to determine the optimal amount needed as input for emulsion-based clonal amplification.

\subsection{Pyrosequencing}

Single effective copies of template species from the DNA library to be sequenced were hybridized to DNA capture beads. Then the immobilized library was resuspended in an amplification solution, and the mixture was emulsified, followed by PCR amplification. The DNA-carrying beads were recovered from the emulsion and enriched after amplification. The second strands of the amplified products were melted, leaving the amplified single-stranded DNA library bound to the beads. Then the sequencing primer was annealed to the immobilized amplified DNA templates. After amplification, a single DNA-carrying bead was placed into each well of a PicoTiterPlate (PTP) device. Simultaneous sequencing with multiple samples on a single PTP (four-region gasket) was used. Then the PTP was inserted into the FLX Genome Titanium sequencer for pyrosequencing [22,23], and sequencing reagent was flowed sequentially over the plate. Information from the PTP wells was captured simultaneously by a camera, and the images were processed in real-time by an onboard computer. Multiplex identifiers were used to specifically tag unique samples in a GS FLX Titanium sequencing run, which were recognized by the GS data analysis software after the sequencing run and provided high confidence for assigning individual sequencing reads to the correct sample. Sequence assembly was performed after sequencing using GS De Novo Assembler software (Roche) to produce contigs and singletons. All sequence data were conformed to references using GS Reference Mapper software (Roche).

\subsection{Discovery of cDNA-SSR Markers}

All contigs and singletons from both transcriptomes were used to mine SSR motifs, and SSR motifs were identified using the ARGOS pipeline program (version 1.46) at the default settings to survey the molecular markers present in the $V$. sativa subsp. sativa accessions. Parameters were designed for identifying perfect di-, tri-, tetra-, penta-, and hexa-nucleotide motifs with a minimum of six repeats. The primer design parameters were set as follows: length range, 18-23 nucleotides with 21 as optimum; PCR product size range, $100-400 \mathrm{bp}$; optimum annealing temperature, $55^{\circ} \mathrm{C}$; and GC content 40-60\%, with $50 \%$ as optimum. Vicia sativa subsp. sativa genomic DNA was extracted from 32 diverse common vetch accessions for cDNA-SSR marker validation using a DNeasy ${ }^{\circledR}$ Plant Mini kit (Qiagen, Valencia, CA, USA), according to the manufacturer's instructions. Fresh leaf tissue from 
each accession was used for each extraction and ground well using liquid nitrogen. DNA was resuspended in $100 \mu \mathrm{L}$ water, and dilutions were made to $10 \mathrm{ng} / \mu \mathrm{L}$ followed by storage at either $-20{ }^{\circ} \mathrm{C}$ or $-80{ }^{\circ} \mathrm{C}$. Randomly selected cDNA-SSR primer pairs were validated experimentally, and forward primers were synthesized by adding the M13 sequence to enable the addition of a fluorescent tail through the PCR amplification process [24]. PCR conditions included a hot-start at $95{ }^{\circ} \mathrm{C}$ for 10 min, followed by 10 cycles at $94{ }^{\circ} \mathrm{C}$ for $30 \mathrm{~s}, 60-50{ }^{\circ} \mathrm{C}$ for $30 \mathrm{~s}$ and $72{ }^{\circ} \mathrm{C}$ for $30 \mathrm{~s}$, followed by 25 cycles at $94{ }^{\circ} \mathrm{C}$ for $30 \mathrm{~s}, 50{ }^{\circ} \mathrm{C}$ for $30 \mathrm{~s}$, and $72{ }^{\circ} \mathrm{C}$ for $30 \mathrm{~s}$, with a final elongation step of $72{ }^{\circ} \mathrm{C}$ for $10 \mathrm{~min}$. PCR products were separated and visualized using the QIAxcel Gel Electrophoresis System (Qiagen).

\subsection{Data Analysis}

The amplified SSR loci were scored for 32 accessions. The total number of alleles (NA), major allele frequency (allele with the highest frequency) $\left(M_{A F}\right)$, observed heterozygosity (counting heterozygocity) $\left(H_{O}\right)$, expected heterozygosity $\left(H_{E}\right)$, number of genotypes $\left(N_{G}\right)$, and polymorphic information content (PIC) were calculated using PowerMarker and GenAlEx (version 6.5) [25].

The expected heterozygosity formula is as follows:

$$
\widehat{D_{l}}=\left(1-\sum_{u=1}^{k} \tilde{p}_{l u}^{2}\right) \text {. }
$$

A closely related diversity measure is the polymorphism information content (PIC) [26]:

$$
\widehat{P I C}_{l}=1-\sum_{u=1}^{k} \tilde{p}_{l u}{ }^{2}-\sum_{u=1}^{k-1} \sum_{v=u+1}^{k} 2 \tilde{p}_{l u}^{2} \tilde{p}_{l v}^{2}
$$

The cluster analysis of 32 accessions was carried out based onunweighted pair group method with arithmetic mean (UPGMA ) phylogenetic and uprooted tree construction, based on the "CS chord 1967" distance method [27] in powermarker

\section{Conclusions}

We developed 65 cDNA-SSR markers, which were used successfully to investigate the genetic diversity among 32 accessions of Vicia stiva subsp. sativa. Considering the relatively high PIC values (0.59 in average), cDNA-SSR in Vicia sativa subsp. sativa is suggested to be an informative genetic marker system, which can also be applied to population genetic studies and marker-assisted selection to mine and accumulate useful alleles to increase the agronomic potential of vetch varieties.

\section{Supplementary Materials}

Supplementary materials can be accessed at: http://www.mdpi.com/1420-3049/18/7/8376/s1. 


\section{Acknowledgments}

This study was carried out with the support of the "Research Program for Agricultural Science \& Technology Development (Project No. PJ008623)”, National Academy of Agricultural Science, Rural Development Administration, Korea.

\section{Conflict of Interest}

The authors declare no conflict of interest.

\section{References}

1. Hueze, V.; Tran, G.; Baumont, R. Common vetch (Vicia sativa). Feedipedia 2011, 12, 53-62.

2. Sullivan, P. Overview of cover crops and green manures. ATTRA 2003, 1-16.

3. Tate, M.; Ennenking, D. Common vetch (Vicia sativa ssp. sativa): Feed or future food. Grain Legumes 2006, 16-17.

4. Tate, M.E.; Enneking, D. A mess of red pottage. Nature 1992, 359, 357-358.

5. Tate, M.E.; Rathjen, J.; Delaere, I.; Enneking, D. Covert trade in toxic vetch continues. Nature 1999, 400, 207-207.

6. Thavarajah, P.; Thavarajah, D.; Premakumara, G.A.; Vandenberg, A. Detection of Common Vetch (Vicia sativa L.) in Lentil (Lens culinaris L.) using unique chemical fingerprint markers. Food Chem. 2012, 135, 2203-2206.

7. Uzun, A.; Gucer, S.; Acikgoz, E. Common vetch (Vicia sativa L.) germplasm: correlations of crude protein and mineral content to seed traits. Plant Foods Hum. Nutr. 2011, 66, 254-260.

8. Firincioglu, H.K.; Unal, S.; Erberkas, E.; Dogruyol, L. Relationships between seed yield and yield components in common vetch (Vicia sativa ssp. sativa) populations sown in spring and autumn in central Turkey. Field Crop. Res. 2010, 116, 30-37.

9. Firincioglu, H.K.; Erbektas, E.; Dogruyol, L.; Mutlu, Z.; Unal, S.; Karakurt, E. Phenotypic variation in autumn and spring-sown vetch (Vicia sativa ssp.) populations in central Turkey. Span. J. Agric. Res. 2009, 7, 596-606.

10. Mutz, K.O.; Heilkenbrinker, A.; Lonne, M.; Walter, J.G.; Stahl, F. Transcriptome analysis using next-generation sequencing. Curr. Opin. Biotechnol. 2012, 24, 22-30.

11. Ronaghi, M. Pyrosequencing sheds light on DNA sequencing. Genome Res. 2001, 11, 3-11.

12. Varshney, R.K.; Close, T.J.; Singh, N.K.; Hoisington, D.A.; Cook, D.R. Orphan legume crops enter the genomics era! Curr. Opin. Plant Biol. 2009, 12, 202-210.

13. Luro, F.L.; Costantino, G.; Terol, J.; Argout, X.; Allario, T.; Wincker, P.; Talon, M.; Ollitrault, P.; Morillon, R. Transferability of the EST-SSRs developed on Nules clementine (Citrus clementina Hort ex Tan) to other Citrus species and their effectiveness for genetic mapping. BMC Genomics 2008, 9, 287-310.

14. Kim, T.S.; Booth, J.G.; Gauch, H.G., Jr.; Sun. Q.; Park. J.; Lee, Y.H.; Lee, K.G. Simple sequence repeats in Neurospora crassa: Distribution, Polymorphism and evolutionary inference. BMC Genomics 2008, 9, 31-48. 
15. Wang, Z.; Fang, B.; Chen, J.; Zhang, X.; Luo, Z.; Huang, L.; Chen, X.; Li, Y. De novo assembly and characterization of root transcriptome using Illumina paired-end sequencing and development of cSSR markers in sweet potato (Ipomoea batatas). BMC Genomics 2010, 11, 726-739.

16. Blanca, J.; Canizares, J.; Roig, C.; Ziarsolo, P.; Nuez, F.; Pico, B. Transcriptome characterization and high throughput SSRs and SNPs discovery in Cucurbita pepo (Cucurbitaceae). BMC Genomics 2011, 12, 104-119.

17. Moe, K.T.; Chung, J.W.; Cho, Y.I, Moon, J.K.; Ku, J.H.; Jung, J.K.; Lee, J.; Park, Y.J. Sequence information on simple sequence repeats and single nucleotide polymorphisms through transcriptome analysis of mungbean. J. Integr. Plant Biol. 2011, 53, 63-73.

18. Kaur, S.; Pembleton, L.W.; Cogan, N.O.; Savin, K.W.; Leonforte, T.; Paull. J.; Materne, M.; Forster, J.W. Transcriptome sequencing of field pea and faba bean for discovery and validation of SSR genetic markers. BMC Genomics 2012, 13, 104-116.

19. Tanase, K.; Nishitani, C.; Hirakawa, H.; Isobe, S.; Tabata, S.; Ohmiya, A.; Onozaki, T. Transcriptome analysis of carnation (Dianthus caryophyllus L.) based on next-generation sequencing technology. BMC Genomics 2012, 13, 292-303.

20. Yang ,T.; Bao, S.Y.; Ford, R.; Jia, T.J.; Guan, J.P.; He, Y.H.; Sun X.L.; Jiang, Hao, J.; Zhang, X.; Zong, X. High-throughput novel microsatellite marker of faba bean via next generation sequencing. BMC Genomics 2012, 13, 602-613.

21. Dangi, R.S.; Lagu, M.D.; Choudhary, L.B.; Ranjekar, P.K.; Gupta, V.S. Assessment of genetic diversity in Trigonella foenum-graecum and Trigonella caerulea using ISSR and RAPD markers. BMC Plant Biol. 2004, 4, 13-24.

22. Elahi, E.; Ronaghi, M. Pyrosequencing: a tool for DNA sequencing analysis. Methods Mol. Biol. 2004, 255, 211-219.

23. Margulies, M.; Egholm, M.; Altman, W.E.; Attiya, S.; Bader, J.S.; Bemben, LA.; Berka, J.; Braverman, MS.; Chen, Y.J.; Chen, Z.; et al. Genome sequencing in microfabricated high-density picolitre reactors. Nature 2005, 437, 376-380.

24. Riley, M. Functions of the gene products of Escherichia coli. Microbiol. Mol. Biol. Rev. 1993, 57, 862-952.

25. Peakall, R.; Smouse, P.E. GenAlEx 6.5: Genetic analysis in Excel. Population genetic software for teaching and research--an update. Bioinformatics 2012, 28, 2537-2539.

26. Botstein, D.; White, R.L.; Skolnick, M.; Davis, R.W.; Construction of a genetic linkage map in man using restriction fragment length polymorphisms. Am. J. Hum. Genet. 1980, 32, 314-331

27. Cavalli-Sforza, L.L.; Edwards, A.W.F.; 1967. Phylogenetic Analysis: Models and Estimation Procedures. Am. J. Hum. Genet. 1967, 19, 233-257.

Sample Availability: Available from the authors Institute.

(C) 2013 by the authors; licensee MDPI, Basel, Switzerland. This article is an open access article distributed under the terms and conditions of the Creative Commons Attribution license (http://creativecommons.org/licenses/by/3.0/). 